\title{
X-ray spectra of compact extragalactic radio sources
}

\section{Citation}

Worrall, D. M., and B. J. Wilkes. 1990. "X-Ray Spectra of Compact Extragalactic Radio Sources." The Astrophysical Journal 360 (September): 396. doi:10.1086/169130.

\section{Published Version}

10.1086/169130

\section{Permanent link}

http://nrs.harvard.edu/urn-3:HUL.InstRepos:32955262

\section{Terms of Use}

This article was downloaded from Harvard University's DASH repository, and is made available under the terms and conditions applicable to Other Posted Material, as set forth at http:// nrs.harvard.edu/urn-3:HUL.InstRepos:dash.current.terms-of-use\#LAA

\section{Share Your Story}

The Harvard community has made this article openly available.

Please share how this access benefits you. Submit a story.

Accessibility 


\title{
X-RAY SPECTRA OF COMPACT EXTRAGALACTIC RADIO SOURCES
}

\author{
D. M. WorRall AND B. J. Wilkes \\ Harvard-Smithsonian Center for Astrophysics \\ Received 1989 August 14 ; accepted 1990 March 12
}

\begin{abstract}
Quasars with similar core-dominant radio properties can be classified by their differences at optical and infrared frequencies. Their X-ray properties, however, might be expected to be similar if, as is commonly believed, the synchrotron self-Compton mechanism relates their radio and X-ray emission. We have compared the $0.1-3.5 \mathrm{keV}$ mean power-law energy spectral indices, $\bar{\alpha}$, as measured with the Einstein Observatory IPC, for three classes: (i) 19 low-polarization flat radio spectrum, core-dominant QSOs (FRS QSOs), (ii) 12 highly polarized QSOs (HPQs), and (iii) 24 radio-selected BL Lac objects. A power-law spectrum with energy index $\alpha$ gives an acceptable fit to the data for each source. We present individual spectral fits for a few sources for which results are not presented elsewhere. The likelihood distribution for $\alpha$ was computed for each source with the normalization and column density of intervening Galactic matter as free parameters. A maximumlikelihood analysis was used to find the mean power-law index, $\bar{\alpha}$, and the standard deviation, $\sigma$, for each class, assuming the intrinsic distribution to be Gaussian.

Results for the HPQs and FRS QSOs are similar, with a best fit of $\bar{\alpha} \approx 0.5$, whereas for the BL Lac objects, $\bar{\alpha} \approx 1.0$. Each of the three samples is consistent with all its objects emitting a spectrum with the same spectral index. This is in contrast to a small sample of X-ray-selected BL Lac objects, which also fit $\bar{\alpha} \approx 1.0$, where IPC observations are inconsistent with a single spectral index. The X-ray spectral difference between HPQs and radio-selected BL Lac objects questions the wisdom of treating these objects together in statistical analyses, and it is a possible difficulty for the hypothesis that BL Lac objects are gravitationally lensed HPQs. We address the implications of our X-ray results for synchrotron self-Compton models.
\end{abstract}

Subject headings: BL Lacertae objects - radiation mechanisms - quasars - X-rays: sources X-rays: spectra

\section{INTRODUCTION}

Most extragalactic radio sources which are core-dominant are identified with BL Lac objects and QSOs. Historically, the QSOs have been divided into two classes depending on the strength of optical polarization: the highly polarized QSOs (those with $p \gtrsim 3 \%$, hereafter HPQs), and the low-polarization flat radio spectrum QSOs (hereafter FRS QSOs). The classification of BL Lac objects has been applied to objects similar to HPQs in their broad-band continuum spectrum and polarization properties but devoid of optical emission lines. Because HPQs and BL Lac objects display strong similarities, they are often discussed jointly as members of the class of "blazars" (Angel and Stockman 1980), and, as individual objects have undergone more detailed study, the boundaries between the three classifications HPQ, FRS QSO, and BL Lac object have become less distinct.

The distinction between HPQs and FRS QSOs has been lessened by high-sensitivity optical and near-infrared observations which have found examples of FRS QSOs with "embedded" polarized components (Impey 1987; Wills 1989). Moreover, simultaneous optical and near-infrared polarization measurements of HPQs (Wills 1989; Wills et al. 1989) have found the polarization to be stronger with increasing wavelength, which has been interpreted as the mixing of a steep polarized component with flatter-spectrum thermal emission and has led to the prediction that many FRS QSOs will be highly polarized in the near-infrared.

The distinction between HPQs and BL Lac objects is lessened by the observation that HPQs sometimes exhibit the optical appearance of BL Lac objects as their emission lines disappear under strong, variable, continuum emission. Even greater ambiguity occurs because objects originally classified as BL Lac objects commonly reveal weak line emission if they are monitored for long enough with sufficient sensitivity. Antonucci et al. (1987) argue (assuming the variable continuum emission is relativistically beamed) that a sensible division of blazars should be on the basis of "isotropic" properties, such as the broad versus narrow nature of the emission lines or the nature of the diffuse radio emission according to the morphological scheme of Fanaroff and Riley (1974), i.e., FR-2 versus FR-1. The faintness of the optical features and the uncertainties in discriminating between FR-2 and FR-1 structures seen end on lead to practical ambiguities, although measurement of the luminosity of diffuse emission may be a reasonable indicator of Fanaroff-Riley nature. In this paper, we have tabulated sources according to their classification in Table 1 of Hewitt and Burbidge (1987), but we discuss separately the effect of the BL Lac objects for which broad emission lines have subsequently been detected or for which the diffuse radio emission may be of type FR-2.

Superluminal motion of radio components, which may be explained by relativistic Doppler beaming, has been detected in BL Lac objects, HPQs, and FRS QSOs. This supports the idea that all core-dominated radio sources are viewed at a small angle to the direction of a jet moving with a bulk relativistic velocity. Knowledge of the X-ray-producing mechanism is crucial to understanding the effects of orientation and beaming. If emitted X-rays are Compton-scattered photons related to the compact radio source through synchrotron selfCompton (SSC) emission, then the combined radio and X-ray 
data can be modeled to give a measure of the bulk relativistic velocity (e.g., Marscher 1983). However, if the X-rays are dominated by synchrotron radiation or any unbeamed emission components, the model calculations at best provide a lower limit to the amount of beaming. HPQs and FRS QSOs appear to exhibit a single $\mathrm{X}$-ray versus $5 \mathrm{GHz}$ radio luminosity correlation (Worrall 1988) with a small enough dispersion that if the radio emission is beamed with a Lorentz factor $\gamma \gtrsim 4$, the $\mathrm{X}$-rays cannot be dominated by an isotropically emitted component. Radio-selected BL Lac objects, although all within a narrow band of luminosity, appear to extend the correlation to lower luminosities, which would support the idea of a continuity of jet phenomenon in BL Lac objects and core-dominant radio QSOs. Similarly, Ledden and O'Dell (1985) find a correlation of $\alpha_{r x}$ (radio to X-ray spectral index) versus $\alpha_{r o}$ (radio to optical spectral index) which is continuous between BL Lac objects and HPQs. We might then expect the X-ray spectra of BL Lac objects, HPQs, and FRS QSOs to be similar, with the strong likelihood that the X-ray emission is dominated by selfCompton scattering of the radio synchrotron emission.

However, the situation is complicated by evidence to suggest that the mixing of unbeamed X-ray emission with beamed emission may be significant for both BL Lac objects and FRS QSOs, but not necessarily to the same extent. The evidence for the X-ray emission in BL Lac objects being more isotropic than the radio emission comes from the finding that X-rayselected BL Lac objects are weaker radio emitters than are radio-selected objects, whereas radio-selected and X-rayselected objects are equally strong X-ray emitters (Stocke et al. 1985; Maraschi et al. 1986; Stocke et al. 1989). For QSOs, the situation is complicated by the fact that radio-quiet QSOs are themselves strong X-ray emitters, although for the same optical luminosity a radio-loud QSO will overproduce X-rays by about a factor of 5 (Ku, Helfand, and Lucy 1980; Zamorani et al. 1981; Worrall et al. 1987). (There is no evidence for radio-quiet BL Lac objects [Stocke et al. 1989].) The Browne and Murphy (1987) analysis of the X-ray relative to radio emission in QSOs of different core dominance within the framework of the "unified scheme" of Orr and Browne (1982) has found that beamed X-rays should outshine unbeamed X-rays from sources we classify as HPQs and FRS QSOs by roughly an order of magnitude. Indications are thus that beaming may be more important in the X-ray emission of the core-dominant rado QSOs than in BL Lac objects, and thus X-ray spectral differences between the classes may be expected.

Alternatively, Ostriker and Vietri (1985) have suggested that a significant fraction of BL Lac objects are HPQs which are gravitationally lensed by pointlike objects in intervening galaxies. In this case, we might expect the X-ray spectra of HPQs and BL Lac objects to be similar.

In this paper we consider the FRS QSO, HPQ, and radioselected BL Lac classes of AGNs separately and compare their $0.1-3.5 \mathrm{keV}$ X-ray spectra to examine the extent that these objects are related. All data are from on-axis (target) observations with the Einstein Observatory imaging proportional counter (IPC). We compare the results for the radio-selected BL Lac objects with those for a small sample of BL Lac objects which were known X-ray sources before their observation with the IPC. Similarities of X-ray spectra between radio-selected and X-ray-selected BL Lac objects might be expected if the $\mathrm{X}$-ray emission is predominantly isotropic. As part of the presentation of our results, for a few sources we show individual spectral fits not presented elsewhere. We examine the influence of assuming that the column density of intervening matter is represented by $21 \mathrm{~cm}$ measurements to the sources, and we show the effect on our results of objects of ambiguous classification.

\section{DATA ANALYSIS}

a) Sample Selection

The lists of Angel and Stockman (1980), Moore and Stockman (1981, 1984), Stockman, Moore, and Angel (1984), and Ledden and O'Dell (1985) were used to search the Einstein Observatory imaging proportional counter (IPC) data bank for HPQs and BL Lac objects which were observed on axis with sufficient net counts $(\gtrsim 80)$ to allow spectral fitting through a good determination of the IPC detector gain during the observation. The objects are listed in Table 1 , where they have been divided into HPQ and BL Lac-type categories according to their designation in Table 1 of Hewitt and Burbidge (1987). The BL Lac objects are further divided by radio versus $\mathrm{X}$-ray selection according to Ledden and O'Dell (1985). Notes to Table 1 indicate which sources listed as BL Lac objects are of questionable classification, due to subsequent detection of faint broad emission lines, or due to detection of diffuse radio emission which may be of Fanaroff-Riley type II. The comparison FRS QSOs are primarily those flat-spectrum, compactstructure, radio-loud QSOs listed in Worrall et al. (1987) which satisfy the requirements for spectral fitting and are not HPQs. The samples comprise 19 FRS QSOs, 12 HPQs, 24 radioselected BL Lac objects, and six X-ray-selected BL Lac objects.

\section{b) Analysis Methods \\ i) X-Ray Spectral Fitting}

On-source and background counts were extracted from a $3^{\prime}$ circle and $5^{\prime}-6^{\prime}$ annulus, respectively, centered on the source position for those pulse-height channels corresponding most closely to an observed energy range of $0.1-3.5 \mathrm{keV}$. A threeparameter model of a single power law with absorption due to the column density of intervening gas in our galaxy (parameterized by normalization, energy index $\alpha$, and $N_{\mathrm{H}}$ ) was convolved with the telescope and detector response and compared with the observed pulse-height distribution using $\chi^{2}$. Best-fit parameter values were found through minimizing $\chi^{2}$. The quality of the fits was sufficiently good that the use of alternative or more complex models was not warranted. Brown and Gould (1970) absorption cross sections were used for consistency with Wilkes and Elvis (1987).

For sources observed more than once, an attempt was made to fit the same model, including normalization, simultaneously to the different observations. In some cases a bad fit resulted from variability of source intensity, but not necessarily spectral shape, and then the weaker observations were not used (see notes to Table 1). An exception was the X-ray-selected BL Lac object $2155-304$, where no single $\alpha$ was consistent with both observations. The two observations of the source have been treated as though they were for separate sources.

In addition to finding the three best-fit parameter values for each source, we investigated uncertainties in $\alpha$ and $N_{\mathrm{H}}$ by plotting the projections of the $\Delta \chi^{2}=2.3,4.6$ and 9.2 surfaces $(68 \%$, $90 \%$ and $99 \%$ confidence for two interesting parameters) onto the $\alpha$ versus $N_{\mathrm{H}}$ plane. Such results for a few sources which are bright enough for the parameter-value constraints to be useful, and for which spectral fits for the reprocessed (Rev 1B) IPC data have not been publised elsewhere, are given in $\S$ III $b$. 
TABLE 1

SAMPLE OBJECTS

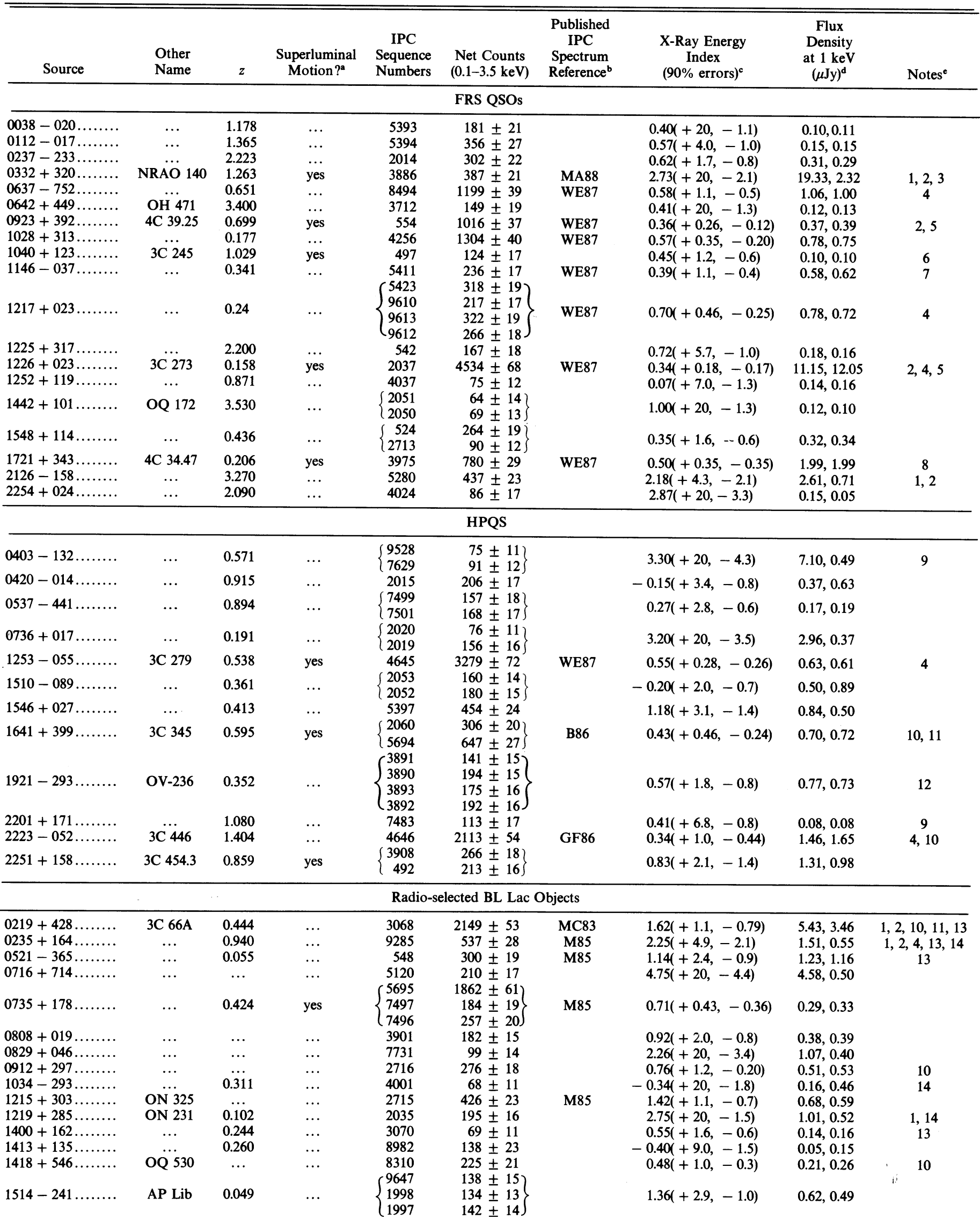


TABLE 1-Continued

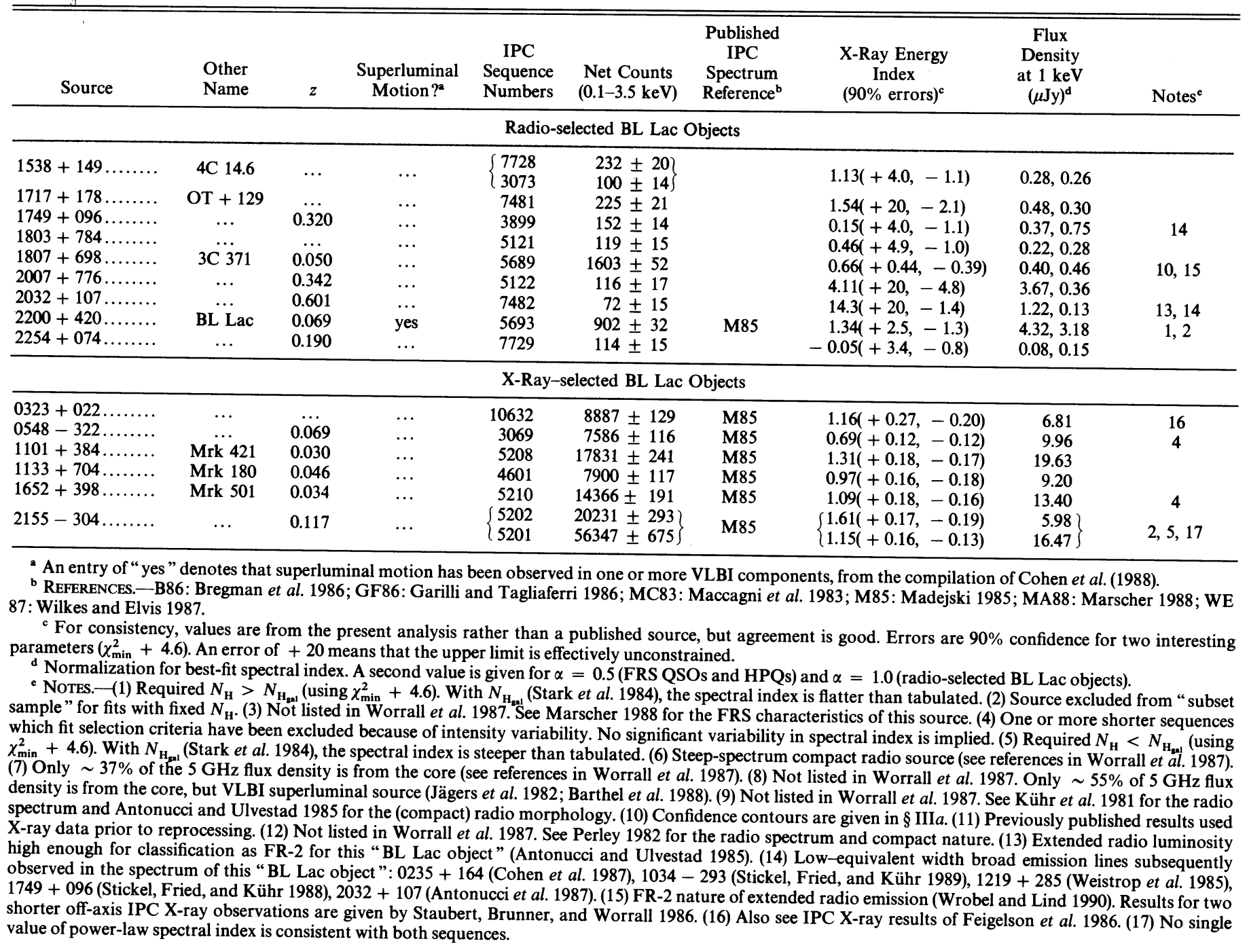

\section{ii) Maximum-Likelihood Analysis}

A model fit of the $n$th source to a power-law model with absorption for a given value of spectral index, $\alpha_{j}$, can be written in terms of the likelihood of $\alpha_{j}, L_{n}\left(\alpha_{j}\right)$, such that, for Gaussian residuals,

$$
L_{n}\left(\alpha_{j}\right)=\exp \left[\frac{-\chi_{n_{\min }}^{2}\left(\alpha_{j}\right)}{2}\right],
$$

where $\chi_{n_{\min }}^{2}$ is determined for the $n$th source by allowing its other spectral parameters to vary. We assume a model in which the intrinsic distribution of spectral index for a sample of sources is distributed by a Gaussian of standard deviation $\sigma$ about a mean value $\bar{\alpha}$, and we wish to evaluate the best-fit values of $\sigma$ and $\bar{\alpha}$. The likelihood of $\sigma$ and $\bar{\alpha}$ for the $n$th source is given by the convolution of $L_{n}\left(\alpha_{j}\right)$ with the Gaussian,

$$
L_{n}(\sigma, \bar{\alpha})=\sum_{j=1}^{J} \frac{\delta \alpha_{j}}{\sigma \sqrt{2 \pi}} \exp \left[\frac{-\left(\alpha_{j}-\bar{\alpha}\right)^{2}}{2 \sigma^{2}}\right] \exp \left[\frac{-\chi_{n_{\min }}^{2}\left(\alpha_{j}\right)}{2}\right] .
$$

To find the best values of $\sigma, \bar{\alpha}$ for the $N$ sources in a sample, we maximize the overall likelihood by minimizing $S=-2 \ln L$, where

$$
\begin{aligned}
S=-2 \sum_{n=1}^{N} \ln \sum_{j=1}^{J} & \frac{\delta \alpha_{j}}{\sigma \sqrt{2 \pi}} \\
& \times \exp \left[\frac{-\left(\alpha_{j}-\bar{\alpha}\right)^{2}}{2 \sigma^{2}}\right] \exp \left[\frac{-\chi_{n_{\min }^{2}}^{2}\left(\alpha_{j}\right)}{2}\right] .
\end{aligned}
$$

In practice, we chose 61 values of $\alpha_{j}$ uniformly spaced at intervals of $\delta \alpha_{j}=0.1$ from -3.0 to +3.0 . Since there are 62 sources listed in Table 1 (treating the two observations of 2155 - 304 separately), we ran a total of 3782 spectral fits, each with two free parameters (normalization and $N_{\mathrm{H}}$ ), to build up a two-dimensional array of $\chi_{n_{\min }^{2}}^{2}\left(\alpha_{j}\right)$ values. We used a prototype of the post reduction off-line software (PROS) X-ray spectral fitting package which runs under IRAF, ${ }^{1}$ with an IRAF "script task" to step through $\alpha_{j}$ and $n$. For each source we constrained

${ }^{1}$ IRAF is distributed by the National Optical Astronomy Observatories, which is operated by the Association of Universities for Research in Astronomy, Inc., under contract to the National Science Foundation. 
$N_{\mathrm{H}}$ to be within a factor of 20 of the appropriate value from Stark et al. (1984), $N_{\mathrm{H}_{\text {gal }}}$, to restrict inclusion of unphysical fits in our maximum-likelihood analysis. $\left(N_{\mathrm{H}_{\mathrm{gal}}}\right.$ and the best-fit value or upper limit for $N_{\mathrm{H}}$ typically differ by a factor of a few.) A separate computer program was written to evaluate the $\bar{\alpha}$ and $\sigma$ values which minimize $S$ (eq. [3]) and to plot the $S=$ $S_{\min }+4.6$ contour which encloses the values of $\bar{\alpha}$ and $\sigma$ which are allowed with $90 \%$ confidence (for two interesting parameters).

Our choice of $N_{\mathrm{H}}$ as a free parameter allows for each source having a variable amount of excess absorption or ultrasoft excess emission. To investigate any systematic degree of either absorption or excess emission for the objects forming a sample, we repeated our analysis with each source's $N_{\mathrm{H}}$ value fixed at that given by Stark et al. (1984), $N_{\mathrm{H}_{\mathrm{gal}}}$. When this is done, in the special case of $\sigma$ tending to zero (i.e., the Gaussian being replaced by a $\delta$-function approximation), the analysis reduces to finding the minimum value of $S\left(\alpha_{j}\right)$, where $S=\sum_{n=1}^{N} \chi_{n_{\min }}^{2}\left(\alpha_{j}\right)$ and $\chi_{n_{\min }}^{2}$ is minimized with respect to normalization. This is mathematically equivalent to finding the best-fit energy index along the line of $N_{\mathrm{H}_{\text {residual }}}=N_{\mathrm{H}}-N_{\mathrm{H}_{\mathrm{gal}}}=0$ in the composite $\chi^{2}$ method applied by Canizares and White (1989). Our maximum-likelihood method has the advantage of testing for a dispersion in the intrinsic distribution of $\alpha$. However, if the dispersion for $N_{\mathrm{H}}=N_{\mathrm{H}_{\mathrm{gal}}}$ can be shown to be consistent with zero, the composite $\chi^{2}$ method is useful for investigating the mean spectral index as a function of excess absorption.

The use of $\chi^{2}$ to test the goodness of fit is compromised where pulse-height bins contain $\lesssim 15$ counts and the approximation of Gaussian residuals is poor. We ascertained that this has negligible influence on our results by reevaluating some of our maximum-likelihood contours excluding such pulse-height channels from our input data.

\section{RESULTS}

\section{a) Power-Law Model Fits to Individual Sources}

All sources fit a three-parameter model of a single power-law with absorption with a probability for exceeding $\chi^{2}$ of $\gtrsim 1 \%$, and all but five sources $(0923+392,1548+114,0420-014$, $0537-441$, and $1807+698$ ) fit with a probability of $\gtrsim 5 \%$. The best-fit power-law spectral energy index for each source and the uncertainty corresponding to $\chi_{\min }^{2}+4.6(90 \%$ for two inter- esting parameters; $97 \%$ for one interesting parameter) are given in Table 1 . The individual sources for which the spectral index is best constrained provide the greatest relative weight in our maximum-likelihood calculations.

Most sources fit a value of $N_{\mathrm{H}}$ which is consistent at $90 \%$ confidence with the value for our galaxy, $N_{\mathrm{H}_{\text {gal }}}$, from Stark $e t$ al. (1984). The nine sources for which this is not true, in that the $\chi^{2}$ value using $N_{\mathbf{H}_{\mathrm{gal}}}$ is larger by at least 4.6 than the $\chi^{2}$ value for the best-fit $N_{\mathrm{H}}$, are listed in Table 2 . Of these nine sources, only three fit $N_{\mathbf{H}}<N_{\mathbf{H}_{\text {gal }}}$. These include two FRS QSOs $(0923+392$, 3C 273) in the list of Wilkes and Elvis (1987), who concluded that $N_{\mathrm{H}}<N_{\mathrm{H}_{\mathrm{gal}}}$ is common for the brightest radio-loud and radio-quiet QSOs and may be due to ultrasoft excess emission, and the X-ray-selected BL Lac object 2155 - 304, for which this effect was attributed by Madejski (1985) to the complex spectral nature below $0.7 \mathrm{keV}$ (Canizares and Kruper 1984). Two FRS QSOs and four radio-selected BL Lac objects fit $N_{\mathrm{H}}>N_{\mathrm{H}_{\mathrm{gal}}}$, which may be indicative of intrinsic absorption or, for the case of the BL Lac objects, convex spectral curvature (Madejski 1985; Barr et al. 1989; Madejski and Schwartz 1989).

\section{b) The Brightest Sources}

In Figures 1 and 2 and Table 3 we present spectral fits for six of our brightest X-ray sources for which fits using the reprocessed (Rev IB) IPC data are not presented elsewhere. For those other sources which yielded sufficient counts for the parameter-value constraints to be useful, our spectral-index results agree within uncertainties with previously published values (see references given in Table 1). Madejski (1985) and Madejski and Schwartz (1988) present IPC spectral fits for some BL Lac object observations which are not listed in Table 1 because they do not meet our strict requirement for on-axis observations.

\section{c) Maximum-Likelihood Results}

$$
\text { i) Average Spectra }
$$

For each sample the summed (composite) $\chi^{2}$ obtained from fitting each of its objects to the three-free parameter model of a single power law with absorption is acceptable $\left[P\left(>\chi^{2}\right) \geq 5 \%\right.$; see Table 4]. The best-fit values for the mean spectral index, $\bar{\alpha}$, and Gaussian standard deviation, $\sigma$, using the maximum-

TABLE 2

Sources Which Give Poor Fits with $N_{\mathrm{H}}=N_{\mathrm{H}_{\mathrm{mal}}}$

\begin{tabular}{|c|c|c|c|c|c|c|}
\hline Source & $\begin{array}{l}\text { Other } \\
\text { Name }\end{array}$ & Class & $N_{\mathrm{H}_{\mathrm{ga} 1}}^{\mathrm{a}}$ & $\Delta \chi^{2 \mathrm{~b}}$ & $\begin{array}{l}\text { Significance } \\
\left(N_{\mathrm{H}}>N_{\mathrm{H}_{\mathrm{ga}}}\right)\end{array}$ & $\begin{array}{l}\text { Significance } \\
\left(N_{\mathrm{H}}<N_{\mathrm{H}_{\mathrm{gal}}}\right)\end{array}$ \\
\hline $0332+320$ & NRAO 140 & FRS QSO & $14.22 *$ & 7.97 & 99.52 & \\
\hline $0923+392 .$. & $4 C 39.25$ & FRS QSO & $1.69 *$ & 8.79 & $\ldots$ & 99.70 \\
\hline $1226+023$. & $3 C 273$ & FRS QSO & 1.83 & 5.39 & $\ldots$ & 97.97 \\
\hline $2126-158$. & & FRS QSO & $4.85^{*}$ & 6.30 & 98.79 & $\ldots$ \\
\hline $0219+428 .$. & $3 \mathrm{C} 66 \mathrm{~A}$ & Radio-selected BL Lac & 7.51 & 8.08 & 99.55 & $\ldots$ \\
\hline $0235+164 .$. & & Radio-selected BL Lac & $7.60^{*}$ & 6.67 & 99.02 & $\ldots$ \\
\hline $1219+285 \ldots$ & ON 231 & Radio-selected BL Lac & 1.81 & 6.70 & 99.04 & $\ldots$ \\
\hline $2200+420 \ldots \ldots \ldots$ & BL Lac & Radio-selected BL Lac & $20.15^{*}$ & 4.85 & 97.24 & \\
\hline $2155-304 \ldots \ldots \ldots$ & $\ldots$ & X-ray-selected BL Lac & 1.73 & $\left\{\begin{array}{r}9.67 \\
21.19\end{array}\right.$ & $\begin{array}{l}\ldots \\
\ldots\end{array}$ & $\begin{array}{r}99.81 \\
>99.999\end{array}$ \\
\hline
\end{tabular}

a Galactic $N_{\mathrm{H}}$, in units of $10^{20}$ atoms $\mathrm{cm}^{-2}$, from other work. An asterisk in this column indicates that the value is from $21^{\prime}$ beam size observations (Elvis, Lockman, and Wilkes 1989), in preference to the larger $\left(\sim 2^{\circ} \times 3^{\circ}\right)$ beam size survey results of Stark et al. 1984. The presence or otherwise of a source in this table is not affected by which $N_{\mathrm{H}}$ results are used. Stark et al. values were used for the maximum-likelihood results for $N_{\mathrm{H}}=N_{\mathrm{H}_{201}}$.

b The value of $\chi_{\left(N_{\mathrm{H}}=N_{\mathrm{Hgal}}\right)}^{2}-\chi_{\min }^{2}$. The two values for source 2155-304 correspond to two separate observations (see Table 1).

${ }^{c}$ Following Avni 1976 for one interesting parameter. 

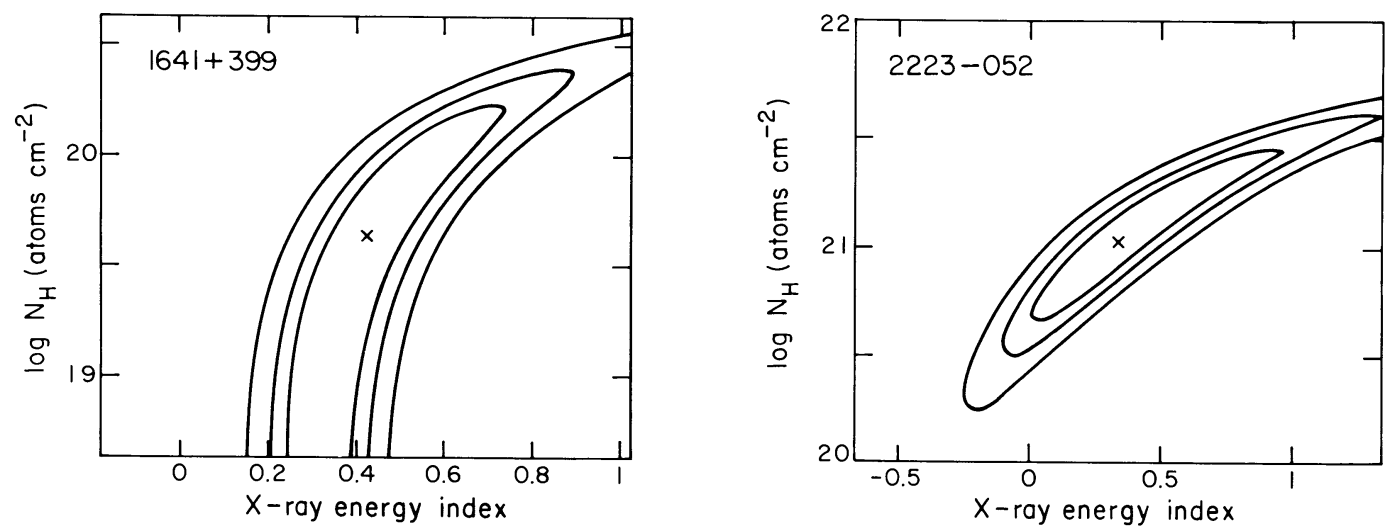

FIG. 1.- Best-fit values for energy spectral index and galactic $N_{\mathrm{H}}$ for a single power-law fit to HPQs $1641+399$ (3C 345) and 2223-052 (3C 446). Contours are $68 \%, 90 \%$, and $99 \%$ confidence for two interesting parameters. See Table 1 for details.

likelihood analysis described in § IIb(ii), are shown in Figure 3. The plotted contours are $90 \%$ confidence for two interesting parameters, or $97 \%$ confidence for one interesting parameter. It is apparent from Figure 3 that the HPQs have a best-fit mean X-ray spectral index similar to the FRS QSOs rather than to the radio-selected BL Lac objects.

ii) Spread in Power-Law Indices

The contours for the FRS QSOs, HPQs, and radio-selected BL Lac objects in Figure 3 are consistent with $\sigma=0$; i.e., all the objects in one of these samples are consistent with a single
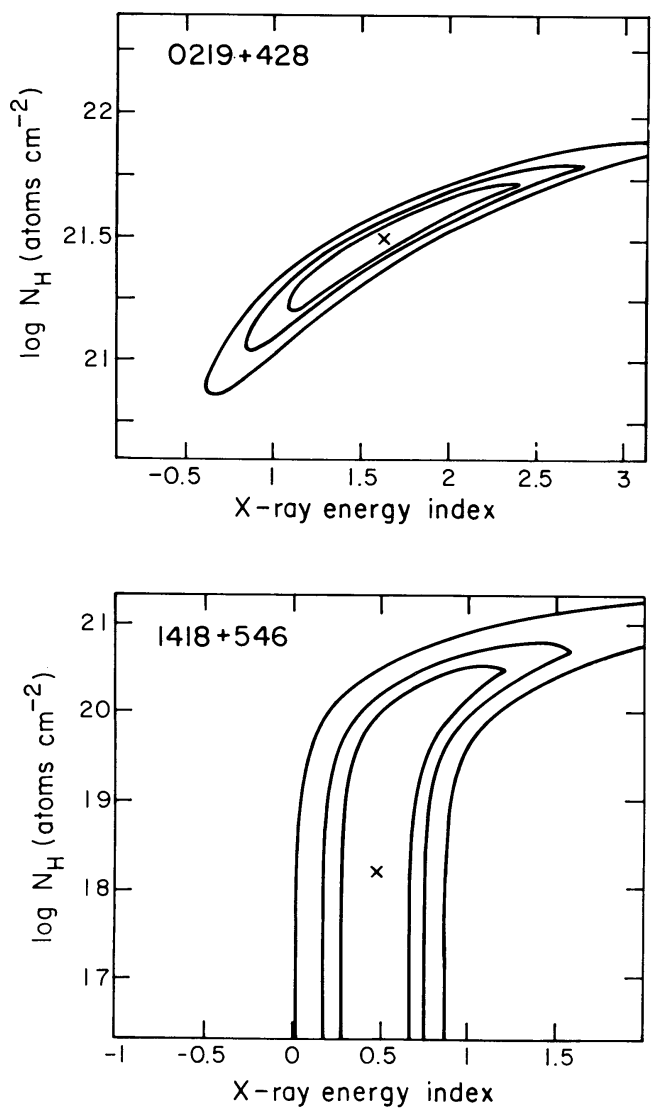

spectral index. This spectral index, $\alpha_{f}$, is 0.5 for the FRS QSOs and HPQs and 1.0 for the radio-selected BL Lac objects. These values are consistent with average values for subsets of our objects presented by Wilkes and Elvis (1987) and Madejski (1985). Our result of $\sigma=0$ is illustrated differently in Table 4, where we see that the composite $\chi^{2}$ value for the two-free parameter model (fixed $\alpha=\alpha_{f}$ ) is acceptable $\left[P\left(>\chi^{2}\right) \geq 5 \%\right]$ for the first three samples. The likelihood distribution of $\alpha$ for the special case of $\sigma=0$ peaks at $\alpha_{f}$ and is shown in Figure 4.

In contrast, X-ray-selected BL Lac objects are inconsistent with a single spectral index. This is illustrated in the lowest
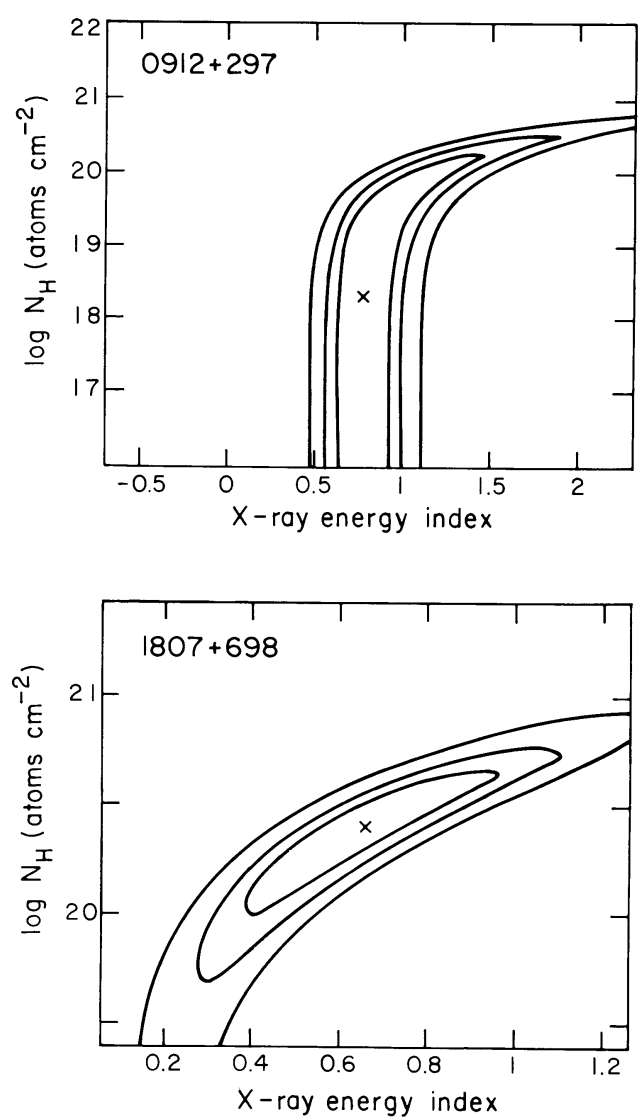

FiG. 2.-Best-fit values for energy spectral index and galactic $N_{\mathrm{H}}$ for a single power-law fit to radio-selected BL Lac objects $0219+428(3 \mathrm{C} 66 \mathrm{~A}), 0912+297$, $1418+546$ (OQ 530), and $1807+698$ (3C 371). Contours are $68 \%, 90 \%$, and $99 \%$ for two interesting parameters. See Table 1 for details. 
TABLE 3

Normalization and GoOdness of Fit for Sources Shown in Figures 1 and 2

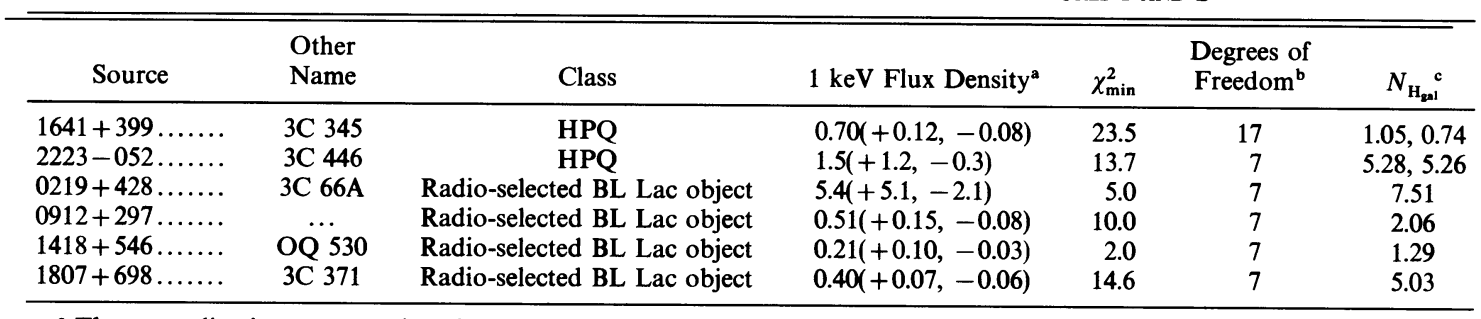

a The normalization, expressed as flux density at $1 \mathrm{keV}$ at the observer, before absorption, in units of $\mu \mathrm{Jy}$. Errors are $90 \%$ confidence for the normalization as the only interesting parameter $\left(\chi_{\min }^{2}+2.7\right.$ with $\alpha$ and $N_{\mathrm{H}}$ free parameters; Avni 1976).

b Degrees of freedom for three parameters $\left(\alpha, N_{\mathrm{H}}\right.$, and normalization).

${ }^{c}$ Galactic $N_{\mathrm{H}}$, in units of $10^{20}$ atoms cm ${ }^{-2}$, from Stark et al. 1984. Where two values are given, the second is from smaller beam size observations of Elvis, Lockman, and Wilkes 1989.
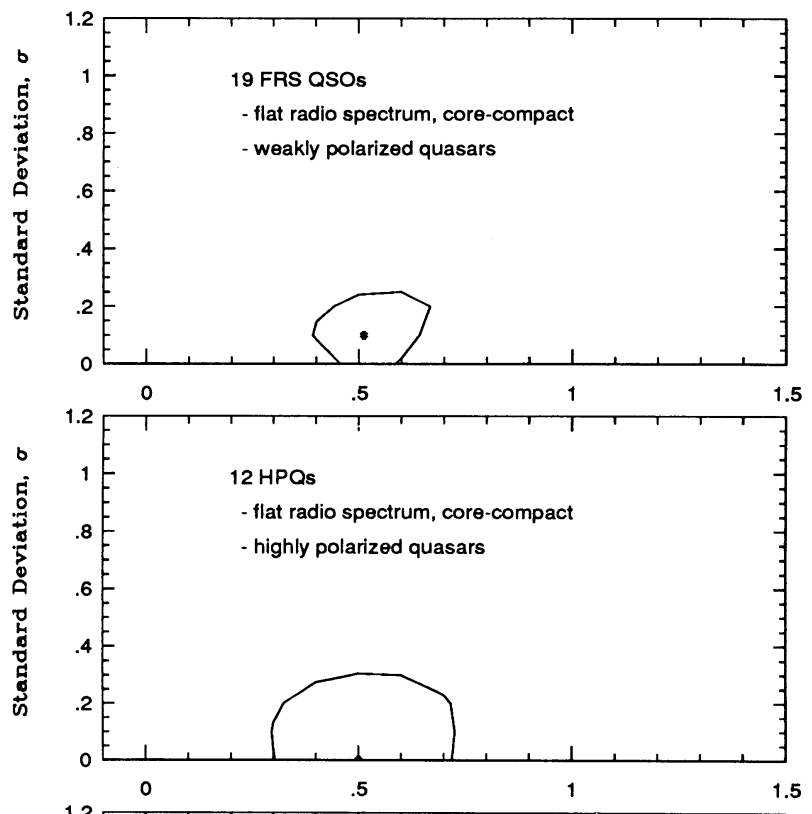
in the case of the radio-selected BL Lac objects, since their detections are poorer (see Fig. 3).

\section{iii) $N_{\mathrm{H}}$}

In Figure 5 we compare results of Figure 3 (solid lines) with those obtained when each source's $N_{\mathrm{H}}$ has been fixed to its $N_{\mathrm{H}_{\mathrm{gal}}}($ dotted lines). We find that $\sigma=0$ now gives a poor fit for all the samples. Since the dispersion for $N_{\mathrm{H}}=N_{\mathrm{H}_{\mathrm{ga}}}$ is not consistent with zero, the composite $\chi^{2}$ method for investigating the mean spectral index as a function of excess absorption (Canizares and White 1989) is not applicable to our sample.
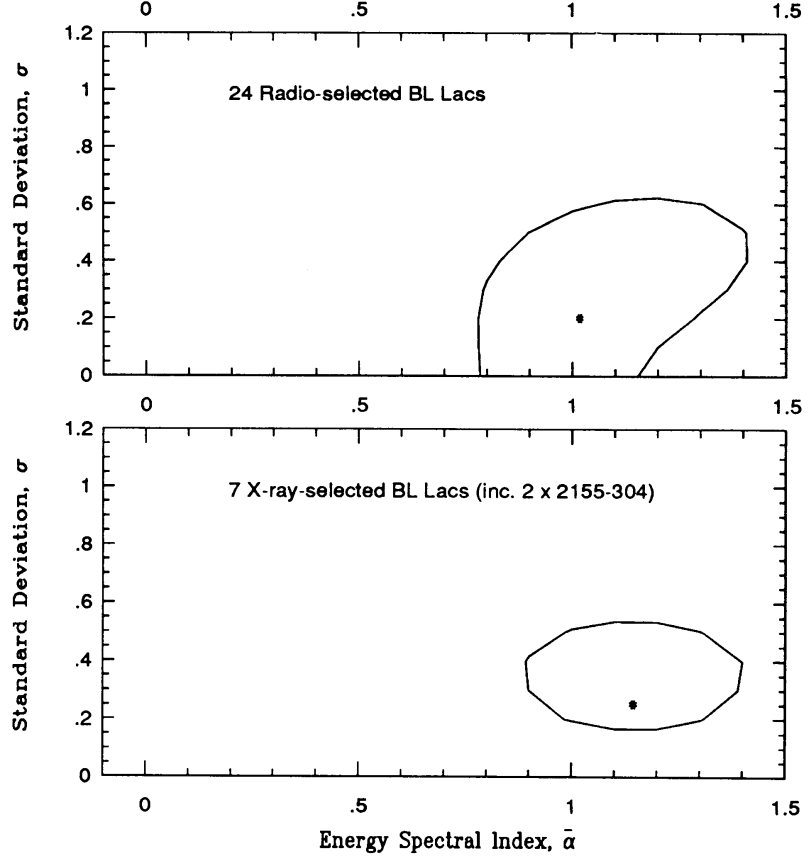

FIG. 3.-The best-fit mean spectral index and Gaussian standard deviation are shown for each sample. The confidence contours correspond to $S_{\min }+4.6$. Results are for each source being fit to an absorbed power law with three free parameters: normalization, $\alpha$, and $N_{\mathrm{H}}$.

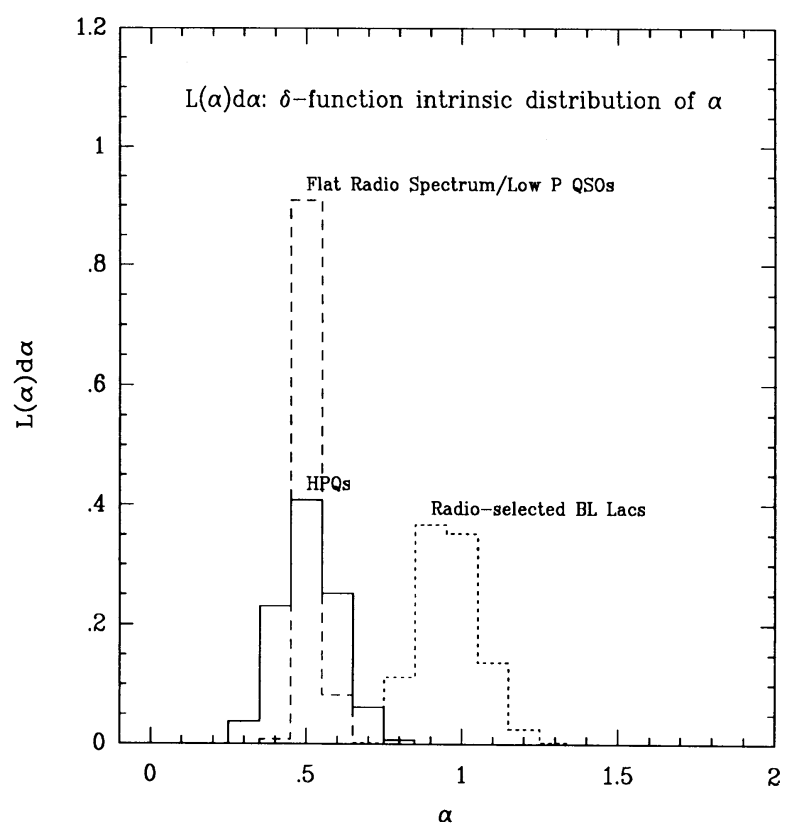

Fig. 4.-Normalized likelihood distributions (not measurement histograms) of $\alpha$ for the special case of each sample having a delta function intrinsic distribution of $\alpha$. Note the separation of the BL Lac objects and the HPQs. 


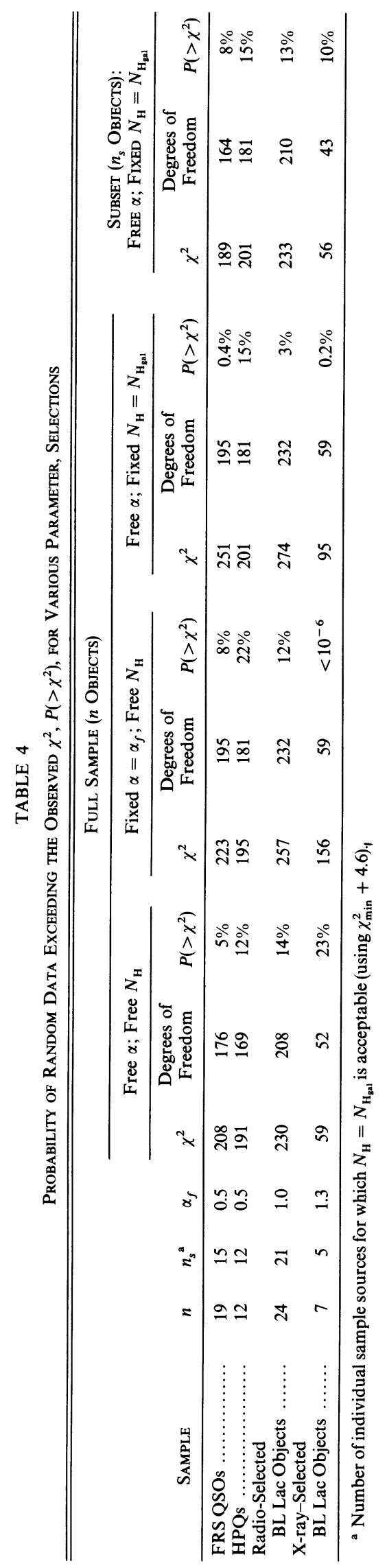


Were it applied, the uncertainty in mean spectral index would be underestimated. ${ }^{2}$

The situation of $N_{\mathrm{H}}=N_{\mathrm{H}_{\mathrm{gal}}}$ is unacceptable statistically for the FRS QSOs, radio-selected BL Lac objects, and X-rayselected BL Lac objects (see Table 4), so that in Figure 5 only the solid-line contours (of Fig. 3) should be used to infer results for $\bar{\alpha}$ for these samples. However, for the FRS QSOs and Xray-selected BL Lac objects the derived $\bar{\alpha}$ is similar regardless of whether or not $N_{\mathrm{H}}$ is fixed to the galactic value. For the radio-selected BL Lac objects, the fact that the average spectral index is flatter for $N_{\mathrm{H}}=N_{\mathrm{H}_{\text {gal }}}$ than for free $N_{\mathrm{H}}$ indicates excess absorption, and indeed the four radio-selected $\mathrm{BL}$ Lac objects which individually fit badly $N_{\mathrm{H}}=N_{\mathrm{H}_{\text {gal }}}$ (Table 2) are all in the direction of $N_{\mathrm{H}}>N_{\mathrm{H}_{\mathrm{gal}}}$. The exclusion of these four sources (dashed line, Fig. 5) does not strongly affect $\bar{\alpha}$, implying that the remaining radio-selected BL Lac objects also have a tendency toward excess absorption. This "excess absorption" may really be a manifestation of the convex spectral curvature reported for the $0.1-10 \mathrm{keV}$ spectra of BL Lac objects (Madejski 1985; Barr et al. 1989; Madejski and Schwartz 1989).

\section{iv) Threshold Effects}

The weight provided by each source to the maximumlikelihood results depends on the degree to which its spectral parameters are constrained. A few strong sources provide as much weight as many weaker sources and, therefore, we have checked that the strong sources are not atypical. We have divided each sample into a "stronger" and a "weaker" subsample which each provide roughly equal weight to the overall sample result, and have analyzed them separately. Results are shown in Figure 6. From the similarities of the solid and dashed lines, we conclude that our results are not unduly biased by a few atypical sources.

\section{v) Sources with Ambiguous Classification}

Some of the sources tabulated by Hewitt and Burbidge (1987) as radio-selected BL Lac objects have subsequently been observed to show weak broad emission lines or diffuse radio emission which is sufficiently luminous to be in the range of Fanaroff-Riley type 2 sources. The classification of such sources as BL Lac objects may be considered questionable (see $\S \mathrm{I})$, and there are nine of them indicated in Table 1. In Figure 7 (upper plot), we show the confidence contours of mean spectral index and Gaussian standard deviation (as in Fig. 3) for the HPQs and the radio-selected BL Lac objects, excluding the nine questionable sources. Although the contours do not completely separate (as they do in Fig. 3), the conclusions are the same. The "questionable" BL Lac objects alone give a contour which is poorly constrained (lower plot, Fig. 7) but which does indicate that the "questionable" BL Lac objects are more similar to the other BL Lac objects in an average X-ray spectrum than they are to the HPQs.

\section{DISCUSSION}

We have compared the $0.1-3.5 \mathrm{keV}$ mean power-law energy spectral indices, $\bar{\alpha}$, for samples of FRS QSOs, HPQs, and radio-selected BL Lac objects. Results for the HPQs and FRS

\footnotetext{
${ }^{2}$ Worrall (1989) shows that there is a similar problem in applying the composite $\chi^{2}$ method to compare the X-ray spectra of radio core-dominated and lobe-dominated QSOs. She concludes that, rather than lobe-dominated QSOs having clearly steeper X-ray spectra than core-dominated QSOs, the confidence regions for the two average X-ray spectra overlap significantly.
}
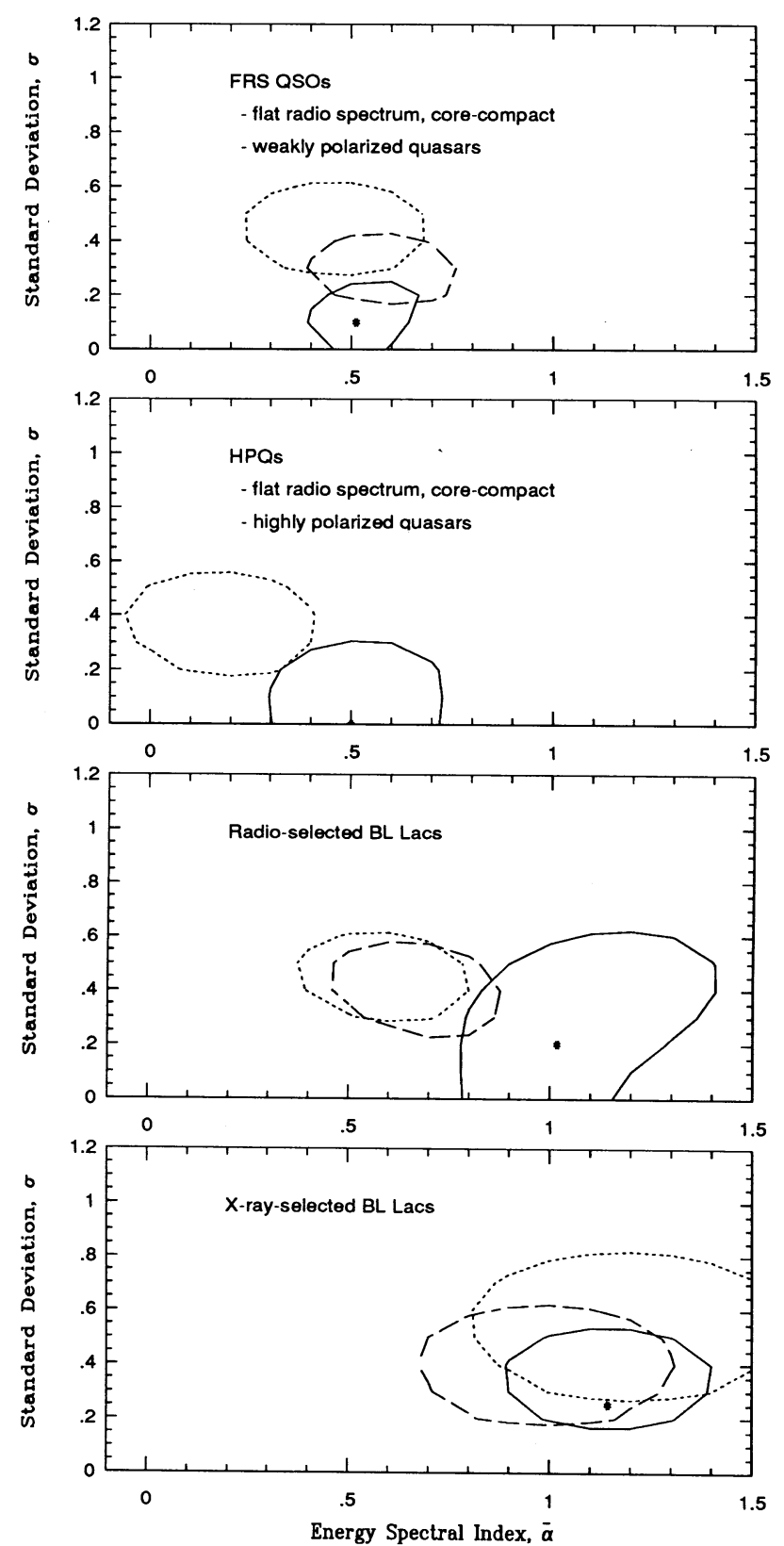

FIG. 5.-Results of Fig. 3 (solid lines) are compared with results for each source being fit to an absorbed power law with $N_{\mathrm{H}}=N_{\mathrm{H}_{\mathrm{i}}}$ and two free parameters: normalization and $\alpha$ (dotted lines). The dashed lines are derived similarly to the dotted lines, except that sources which individually give a poor fit to $N_{\mathrm{H}}=N_{\mathrm{H}_{\text {ont }}}$ (listed in Table 2) have been excluded. The situation $N_{\mathrm{H}}=$ $N_{\mathrm{H}_{\mathrm{g} a 1}}$ is unacceptable statistically for the FRS QSOs, radio-selected BL Lac objects, and X-ray-selected BL Lac objects (see Table 4), for which only the solid line contours should be used to infer results for $\bar{\alpha}$ (although very wrong conclusions would be drawn only in the case of the radio-selected BL Lac objects). Comparison of the dotted and solid lines for the radio-selected BL Lac objects can be used to infer that their average spectrum curves down to low energies (see § III $c$ [iii]).

QSOs are similar, with a best fit of $\bar{\alpha} \approx 0.5$, whereas for the BL Lac objects, $\bar{\alpha} \approx 1$.0. These X-ray spectral differences question the wisdom of treating these objects together, without distinction, in statistical analyses. In particular, our results do not support the suggestion of Ostriker and Vietri (1985) that a significant fraction of BL Lac objects are HPQs which are 

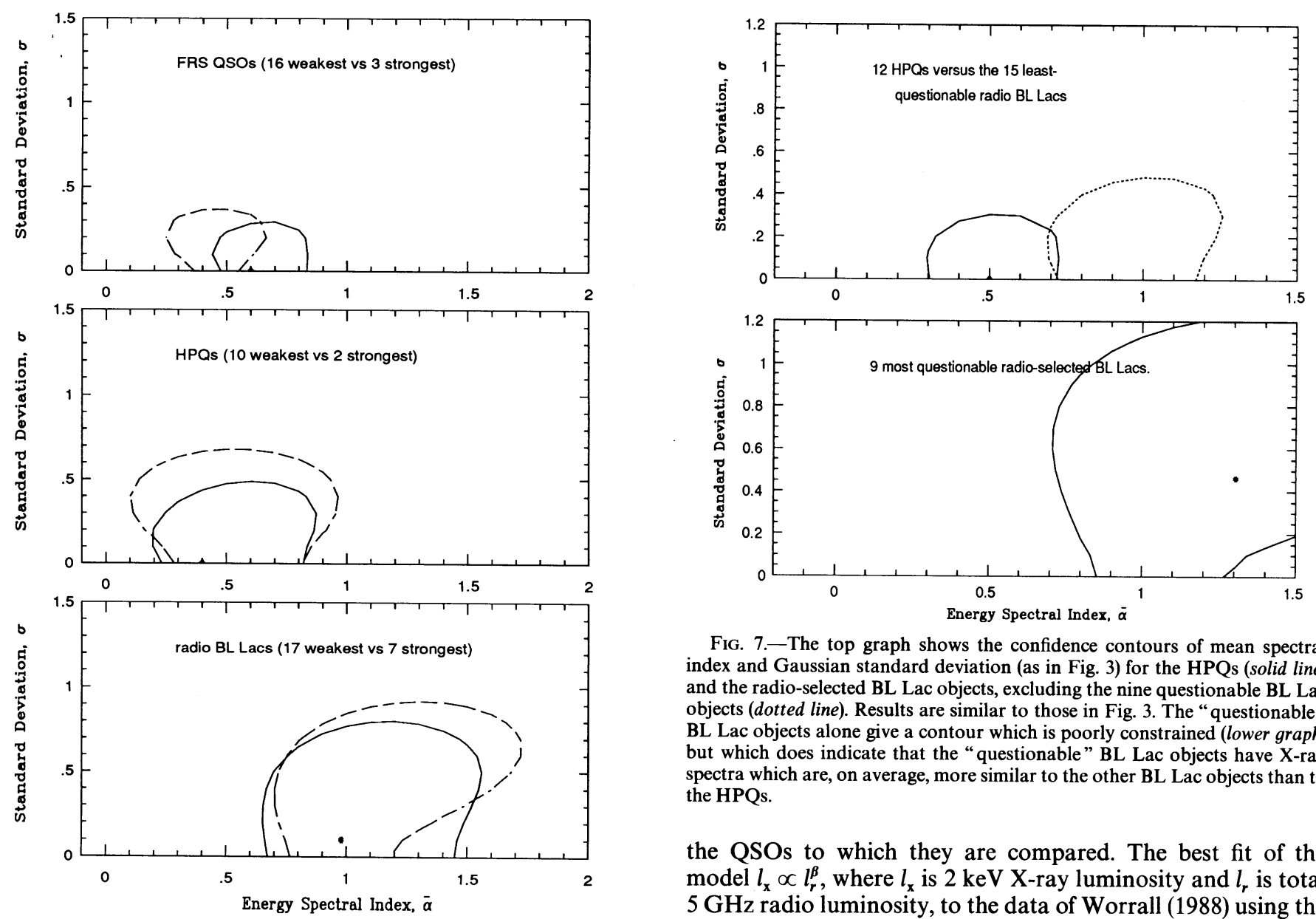

Fig. 7.-The top graph shows the confidence contours of mean spectral index and Gaussian standard deviation (as in Fig. 3) for the HPQs (solid line) and the radio-selected BL Lac objects, excluding the nine questionable BL Lac objects (dotted line). Results are similar to those in Fig. 3. The "questionable" BL Lac objects alone give a contour which is poorly constrained (lower graph) but which does indicate that the "questionable" BL Lac objects have X-ray spectra which are, on average, more similar to the other BL Lac objects than to the HPQs.

the QSOs to which they are compared. The best fit of the model $l_{\mathrm{x}} \propto l_{r}^{\beta}$, where $l_{\mathrm{x}}$ is $2 \mathrm{keV} \mathrm{X-ray} \mathrm{luminosity} \mathrm{and} l_{r}$ is total $5 \mathrm{GHz}$ radio luminosity, to the data of Worrall (1988) using the method of Avni et al. (1980), gives a value for $\beta$ of $0.82 \pm 0.08$

FIG. 6.-Results where significance of detection has been used to divide each sample into two subsamples giving roughly equal weight to the results of Fig. 3. Solid lines are for the weakest sources, and dashed lines are for the strongest. Note that the few sources most weighting our results do not appear to be atypical of their samples. The strongest sources are FRS QSOs: $1226+023,0923+392,1028+313$; HPQs: $1253-055,2223-052$; radioselected BL Lac objects: $0219+428,1807+698,0735+178,2200+420$ $0235+164,1215+303,1514-241$.

gravitationally lensed by pointlike objects in intervening galaxies. Lensing can only affect the spectral shape of the emission if more than one component is present and only one component is lensed. This is highly unlikely for the $\mathrm{X}$-ray emission of compact extragalactic radio sources for which models (see, e.g., Ghisellini, Maraschi, and Treves 1985), as supported by $\mathrm{X}$-ray variability arguments, suggest $\mathrm{X}$-ray source sizes less than a parsec.

The difference in mean spectral index between the coredominated radio QSOs (i.e., the FRS QSOs and the HPQs) and the radio-selected BL Lac objects may be understood in terms of a different mixing of beamed and unbeamed X-ray emission between the classes. Evidence for this was presented in $\S \mathrm{I}$. Since the hypothesis is that beamed emission is more dominant in the QSOs than in the BL Lac objects, our results are satisfied if the beamed component has a flatter spectral index than the unbeamed radiation. It then remains for us to understand the correlations of spectral luminosities and luminosity ratios which appear to be shared by the BL Lac objects and the QSOs, also referred to in $\S \mathrm{I}$. To do so, we note that the BL Lac objects are at lower redshift, i.e., lower luminosity, than (90\% error; see Worrall 1989). This implies that less luminous objects produce more $\mathrm{X}$-rays per unit radio luminosity than do higher luminosity objects. Similarly, the correlations of Ledden and O'Dell (1985) show $\alpha_{r x}$ to be flattest for the lowest redshift (and luminosity) objects. (Lower redshift [and luminosity] objects also tend to have radio to X-ray or ultraviolet spectra which are more broadly "humped " [Ledden and O'Dell 1985; Jones, Rudnick, and Landau 1986].) For illustration, we hypothesize that $l_{x}$ is composed of a beamed inverse Compton component, $l_{x_{i \mathrm{C}}}$, and an isotropic component, $l_{x_{0}}$, that $l_{x_{i \mathrm{C}}}$ is $90 \%$ of $l_{x}$ for the QSOs, and that the ratio $l_{x_{i}} / l_{r}$ is a constant. Comparing the mean value of $\log l_{r}$ for the radio-selected BL Lac objects in Table 1 with that for the HPQs and FRS QSOs (using radio data given in Worrall et al. 1987, Ledden and O'Dell 1985, or as referenced in the notes to Table 1) we find that the radio-selected BL Lac objects are 25 times less luminous in $l_{r}$ than are the QSOs. This observation, together with our assumptions and $l_{x} \propto l_{r}^{0.82}$, then gives that $l_{x_{0}}$ and $l_{x_{i C}}$ contribute equally to $l_{x}$ for the radio-selected BL Lac objects. Thus the correlations have sufficient luminosity dependence to allow a different mix of beamed and unbeamed X-rays between coredominated radio QSOs and radio-selected BL Lac objects.

Figure 8 shows that for the QSOs and radio-selected BL Lac objects the X-ray spectral index, $\alpha_{x}$, is flatter than the implied spectral index between the optical and X-ray bands, $\alpha_{o x}$. For the QSOs, since we have inferred that $l_{x_{i \mathrm{C}}}$ dominates $l_{x}$, our result is equivalent to $\alpha_{x_{i C}}<\alpha_{o x}$. For the BL Lac objects, our result may be caused by one or both of $\alpha_{x_{i C}}$ and $\alpha_{x_{o}}$ being less 

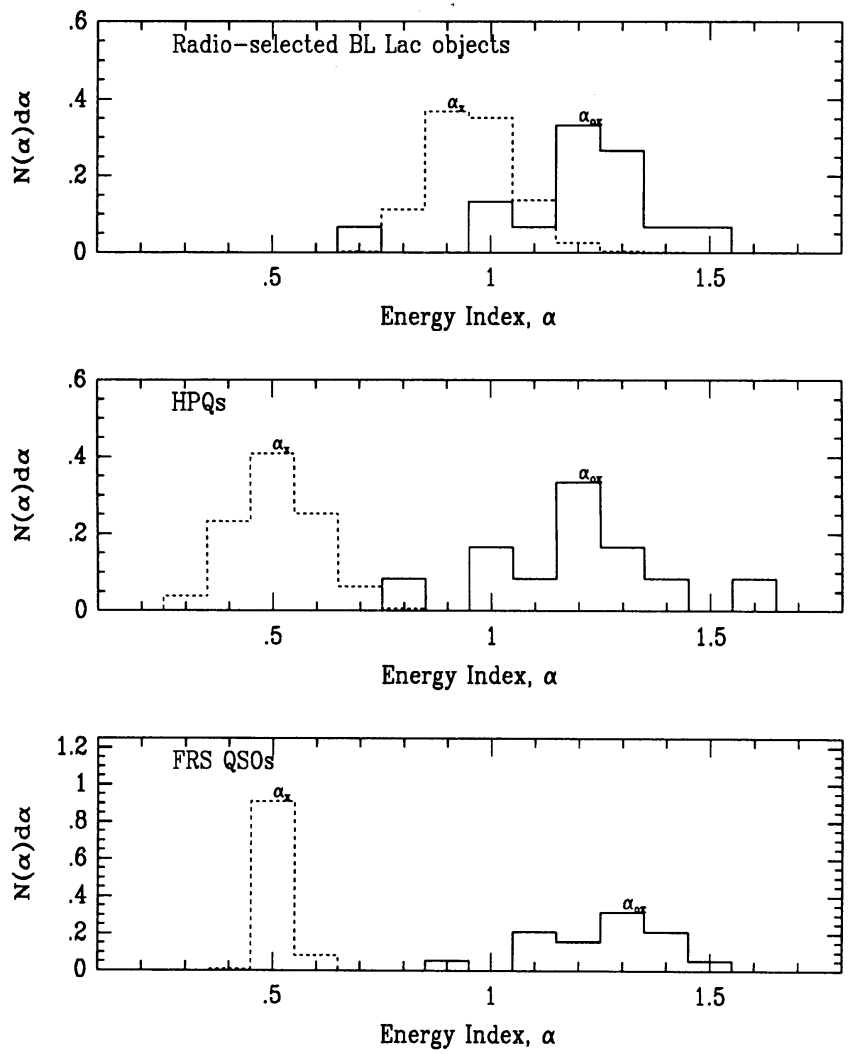

FIG. 8.-Solid lines are normalized histograms of $\alpha_{o x}$, the effective $2500 \AA$ to $2 \mathrm{keV}$ spectral index defined in the rest frame of the source. Results of Fig. 4 (dotted lines) are shown for comparison. All three samples display spectral flattening in the X-ray band. Note that the $\alpha_{o x}$ distributions are not representative of those of the parent populations. Our selection effect of requiring sufficient X-ray counts for spectral fitting biases $\alpha_{o x}$ toward low values.

than $\alpha_{o x}$. However, it is attractive to assume that here also the $\mathrm{X}$-ray spectral flattening is dominated by the inverse Compton component. The reason is that this would allow the optical emission to extrapolate to $l_{x_{0}}$ for both the BL Lac objects and the QSOs and, since the extrapolation would be flatter for the BL Lac objects than for the QSOs, it may help to explain the observation that the radio to ultraviolet spectrum is more broadly " humped" for the BL Lac objects than for the QSOs.
Our example above assumed the same $l_{x_{i} /} l_{r}$ for BL Lac objects and HPQs, i.e., similar intrinsic brightness temperatures for the radio-emitting regions. Madau, Ghisellini, and Persic (1987) have noted that the distribution of relativistic bulk Doppler factors, $\delta$, for BL Lac objects has a lower mean than that for HPQs. However, since the intrinsic brightness temperature is inversely proportional to both $\delta$ and $\theta^{2}$, where $\theta$ is the size of the emitting region, a difference in $\delta$ may be at least partially balanced by larger emitting regions for the $\mathrm{BL}$ Lac objects, for which there is some evidence in the data of Madau, Ghisellini and Persic.

The model described by Maraschi (1988), based on work by Ghisellini, Maraschi, and Treves (1985) (see also Madejski and Schwartz 1989), gives a mixture of isotropic and beamed X-ray emission for BL Lac objects. In this model, the Lorentz factor, $\gamma$, increases with distance from the source, such that the isotropic emission is from low- $\gamma$ (isotropic) inner source regions. If the isotropic X-ray emission in lobe-dominated radio-loud QSOs argued for by Browne and Murphy (1987) comes from a similar site, we might expect at least partial obscuration by emission-line gas in a plane perpendicular to the radio axis (see e.g., Wills and Wills 1985).

In conclusion, the difference we find between the average X-ray spectra of radio-selected BL Lac objects and coredominated radio QSOs may be consistent with a different mixing of isotropic and beamed X-ray emission between the classes. The intrinsic brightness temperatures of the radioemitting regions may be similar for the BL Lac objects and the QSOs, leading to a similar $l_{x_{i} \mathrm{C}} l_{r}$. Only the BL Lac objects reveal excess, steeper spectrum, isotropic X-ray emission which may also be enhancing their lower frequency (optical) emission.

We thank Robert Antonucci, John Wardle, and Joan Wrobel for helpful discussions and Mark Birkinshaw for a major contribution to our analysis methods, to which Andy Pollock added helpful suggestions. We are particularly grateful to Adam Szczypek for writing the prototype PROS/IRAF $\mathrm{X}$-ray spectral fitting package which was used in this project, and to Martin Elvis for his contributions to its specifications. We gratefully acknowledge support from NASA contract NAS8-30751.
Angel, J. R. P., and Stockman, H. S. 1980, Ann. Rev. Astr. Ap., 18, 321.

Antonucci, R. R. J., Hickson, P., Miller, J. S., and Olszewski, E. W. 1987, A.J., 93, 785 .

Antonucci, R. R. J., and Ulvestad, J. S. 1985, Ap. J., 294, 158.

Avni, Y. 1976, Ap. J., 210, 642.

Avni, Y., Soltan, A., Tananbaum, H., and Zamorani, G. 1980, Ap. J., 238, 800.

Barr, P., Giommi, P., Pollock, A., Tagliaferri, G., Maccagni, D., and Garilli, B. 1989, in Active Galactic Nuclei, ed. D. E. Osterbrock and J. Miller (Dordrecht: Kluwer), p. 191.

Barthel, P. D., Schilizzi, R. T., Miley, G. K., Preuss, E., and Hooimeyer, J. R. 1988, in The Impact of VLBI on Astrophysics and Geophysics, ed. M. J. Reid and J. M. Moran (Dordrecht: Reidel), p. 17

Bregman, J. N., et al. 1986, Ap. J., 301, 708 (B86)

Brown, R. L., and Gould, R. J. 1970, Phys. Rev. D, 2, 2252

Browne, I. W. A., and Murphy, D. W. 1987, M.N.R.A.S., 226, 601.

Canizares, C. R., and Kruper, J. 1984, Ap. J. (Letters), 278, L99.

Canizares, C. R., and White, J. L. 1989, Ap. J., 339, 27.

Cohen, M. H., Barthel, P. D., Pearson, T. J., and Zensus, J. A. 1988, A p. J., 329, 1.

Cohen, R. D., Smith, H. E., Junkkarinen, V. T., and Burbidge, E. M. 1987, Ap. J., 318, 577.

\section{REFERENCES}

Elvis, M., Lockman, F. J., and Wilkes, B. J. 1989, A.J., 97, 777.

Fanaroff, B. L., and Riley, J. M. 1974, M.N.R.A.S., 167, 31 p.

Feigelson, E. D., et al. 1986, Ap. J., 302, 337

Garilli, B., and Tagliaferri, G. 1986, Ap. J., 301, 703 (GF86).

Ghisellini, G., Maraschi, L., and Treves, A. 1985, Astr. Ap., 146, 204.

Hewitt, A., and Burbidge, G. 1987, Ap. J., Suppl., 63, 1.

Impey, C. 1987, in Superluminal Radio Sources, ed. J. A. Zensus and T. J. Pearson (Cambridge: Cambridge University Press), p. 233.

Jägers, W. J., van Breugel, W. J. M., Miley, G. K., Schilizzi, R. T., and Conway, R. G. 1982, Astr. Ap., 105, 278

Jones, T. W., Rudnick, L., and Landau, R. 1986, in Continuum Emission in Active Galactic Nuclei, ed. M. L. Sitko (Tucson: Kitt Peak National Observatory), p. 122.

Ku, W. H.-M., Helfand, D. J., and Lucy, L. B. 1980, Nature, 288, 323.

Kühr, H., Witzel, U., Pauliny-Toth, I. I. K., and Nauber, U. 1981, Astr. Ap. Suppl., 45, 367.

Ledden, J. E., and O’Dell, S. L. 1985, Ap. J., 298, 630.

Maccagni, D., Maraschi, L., Tanzi, E. G., Tarenghi, M., and Chiappetti, L. 1983, Ap. J., 273, 75 (MC83).

Madau, P., Ghisellini, G., and Persic, M. 1987, M.N.R.A.S. 224, 257.

Madejski, G. M. 1985, Ph.D. thesis, Harvard University (M85). 
Madejski, G. M., and Schwartz, D. A. 1988, Ap. J., 330, 776. 1989, in BL Lac Objects, ed. L. Maraschi, T. Maccacaro, and M.-H. Ulrich (Berlin: Springer), p. 267.

Maraschi, L. 1988, in The Physics of Compact Objects, ed. N. E. White and L. G. Filipov (Oxford: Pergamon), p. 85.

Maraschi, L., Ghisellini, G., Tanzi, E. G., and Treves, A. 1986, Ap. J., 310, 325 Marscher, A. P. 1983, Ap. J., 264, 296. 1988, Ap. J 334, 552(MA88)

Moore, R. L., and Stockman, H. S. 1981, Ap. J., 243, 60.

. 1984, Ap. J., 279, 465.

Orr, M. J. L., and Browne, I. W. A. 1982, M.N.R.A.S., 200, 1067

Ostriker, J. P., and Vietri, M. 1985, Nature, 318, 446.

Perley, R. A. 1982, A.J., 87, 859.

Stark, A. A., Heiles, C., Bally, J., and Linke, R. 1984, Bell Labs, privately distributed magnetic tape.

Staubert, R., Brunner, H., and Worrall, D. M. 1986, Ap. J., 310, 694.

Stickel, M., Fried, J. W., and Kühr, H. 1988, Astr. Ap., 191, L16. 1989, Astr. Ap. Suppl. $80,103$.

Stocke, J. T., Liebert, J., Schmidt, G. D., Gioia, I. M., Maccacaro, T., Schild, R. E., Maccagni, D., and Arp, H. C. 1985, Ap. J., 298, 619.

Stocke, J. T., Morris, S. L., Gioia, I. M., Maccacaro, T., Schild, R. E., and Wolter, A. 1989, in BL Lac Objects, ed. L. Maraschi, T. Maccacaro, and M.-H. Ulrich (Berlin: Springer), p. 242.
Stockman, H. S., Moore, R. L., and Angel, J. R. P. 1984, Ap. J. 279, 485

Weistrop, D., Schaffer, D. B., Hintzen, P., and Romanishin, W. 1985, Ap. J. 292,614

Wilkes, B. J., and Elvis, M. 1987, Ap. J., 323, 243 (WE 87).

Wills, B. J. 1989, in BL Lac Objects, ed. L. Maraschi, T. Maccacaro, and M.-H. Ulrich (Berlin: Springer), p. 109.

Wills, B. J., Hough, J. H., Bailey, J. A., Wills, D., and Ballard, K. 1989, in preparation.

Wills, B. J., and Wills, D. 1985, in Quasars, ed. G. Swarup and V. K. Kapahi (Dordrecht: Reidel), p. 215.

Worrall, D. M. 1988, in Active Galactic Nuclei, ed. H. R. Miller and P. J. Wiita (Berlin: Springer), p. 248.

p. 1989, in Proc. 23rd ESLAB Symposium, (Noordwijk: ESA SP-296), p. 719 .

Worrall, D. M., Giommi, P., Tananbaum, H., and Zamorani G. 1987, Ap. J., 313, 596 .

Wrobel, J. M., and Lind, K. R. 1990, Ap. J., 348, 135.

Zamorani, G., et al. 1981, Ap. J., 245, 357.

B. J. Wilkes and D. M. Worrall: Harvard-Smithsonian Center for Astrophysics, 60 Garden Street, Cambridge, MA 02138 